\title{
The Diagonalization Paradox Expanded
}

Ron Ragusa ${ }^{1}$

${ }^{1}$ Affiliation not available

July 1, 2020

\begin{abstract}
In 1891 Georg Cantor published his Diagonal Method which, he asserted, proved that the real numbers cannot be put into a one-to-one correspondence with the natural numbers. In this paper we will see how by varying the initial conditions of Cantor's proof we can use the diagonal method to produce a one-to-one correspondence between the set of natural numbers and the set of infinite binary decimals in the interval $(0,1)$. In the appendix we demonstrate that using the diagonal method recursively will, at the limit of the process, fully account for all the infinite binary decimals in $(0,1)$. The proof will cement the one-to-one correspondence between the natural numbers and the infinite binary decimals in $(0,1)$.
\end{abstract}

\section{Hosted file}

The_Diagonalization_Paradox_Expanded(4).pdf available at https://authorea.com/users/266989/ articles/464858-the-diagonalization-paradox-expanded 